\title{
Modeling the dynamic fracture in concrete: an eigensoftening meshfree approach
}

\author{
Pedro Navas ${ }^{a}$, Rena C. $\mathrm{Yu}^{a, 1}, \mathrm{Bo} \mathrm{Li}^{b}$ and Gonzalo Ruiz ${ }^{a}$ \\ ${ }^{a}$ E. T. S. de Ingenieros de Caminos, C. y P., Universidad de Castilla-La Mancha \\ 13071 Ciudad Real, Spain \\ ${ }^{b}$ Dept. of Mechanical and Aerospace Engineering, Case Western Reserve University, \\ Cleveland, Ohio 44106, USA \\ http://www.sciencedirect.com/science/article/pii/S0734743X1730739X
}

\footnotetext{
${ }^{1}$ Corresponding author: rena@uclm.es 


\title{
Modeling the dynamic fracture in concrete: an eigensoftening meshfree approach
}

\author{
Pedro Navas ${ }^{a}$, Rena C. $\mathrm{Yu}^{a, 1}, \mathrm{Bo} \mathrm{Li}^{b}$ and Gonzalo Ruiz ${ }^{a}$ \\ ${ }^{a}$ E. T. S. de Ingenieros de Caminos, C. y P., Universidad de Castilla-La Mancha \\ 13071 Ciudad Real, Spain \\ ${ }^{b}$ Dept. of Mechanical and Aerospace Engineering, Case Western Reserve University, \\ Cleveland, Ohio 44106, USA
}

\begin{abstract}
The objective of this work is twofold. First, we develop an eigensoftening algorithm to capture the gradual process of failure in materials with a softening behavior as an extension of the eigenerosion approach. Both methods are validated against the physical measurements in three-point bending tests of concrete with a drop-weight device, including impact and reaction forces, loading-line displacements as well as strain histories from gauges. The comparison shows that the eigenerosion algorithm significantly overestimates the tensile stresses and the strain peaks, while it captures the forces and crack pattern accurately. Predictions made by the proposed eigensoftening algorithm agree very well with experimental results in all aspects. Second, the energy evolution and partition in the beam predicted by the eigensoftening algorithm at various impact rates is analyzed to quantify the rate dependent fracture properties of concrete. It is demonstrated that, at impact loading conditions, the area below the reactiondeflection curve is much larger than the dissipated fracture energy.
\end{abstract}

Keywords: OTM, eigenerosion, eigensoftening, drop-weight machine

\footnotetext{
${ }^{1}$ Corresponding author: rena@uclm.es
} 


\section{Introduction}

The rate effect on fracture propagation in concrete with or without reinforcements has been the centre of interest for the last three decades, from both experimental and numerical standpoints. Within the framework of finite element

5 methods, typical techniques to capture crack propagation vary from employing cohesive approaches $[1,2]$ by adaptively inserting cohesive elements $[3,4,5]$ at solid element boundaries to adopting crack band theory [6] or handling arbitrary crack paths by level set representation of the fracture surface [7, 8]. Within the scenario of meshfree methods, the visibility criterion [9] or particle erosion $[10,11,12,13]$ have been opted for to reproduce fracture propagation. In the current work, we attempt to model the dynamic fracture propagation in high-strength concrete (HSC) employing the optimal transportation meshfree (OTM) approximation scheme $[14,15,12,13]$. Such a scheme is chosen for its numerous advantages such as the exact mass transport, the satisfaction of the continuity equation, exact linear and angular momentum conservation. Since the deformation and velocity fields are interpolated from nodal values using shape functions based on the principle of maximum entropy, the Kroneckerdelta property at the boundary makes it possible for the direct imposition of essential boundary conditions.

Regarding fracture, we first assess the applicability of an eigenerosion algorithm to quasi-brittle materials such as concrete. The eigenerosion approach to brittle fracture was developed by Pandolfi et al. [11, 12]. They approximate the crack set by means of eigen-deformations, which enable the material to develop displacement jumps at no cost of local elastic energy. The erosion of the material means that each material point can be either intact or be completely failed or eroded and has no load bearing capacity. Even though the method has been successfully applied to dynamic fragmentation of metals, quantitative 
validations, such as stress or strain levels near or at the crack set compared to experimental measures, are still lacking.

Having in mind that the eigenerosion approach was intended for perfectly brittle fracture, an eigensoftening concept is developed for quasi-brittle materials. This is engineered for the gradual rather than abrupt dissipation of the fracture energy. In other words, the material softens in a progressive manner until its complete failure (i.e. the formation of a stress-free crack). This is equivalent to the crack band model [6], since energy dissipation is through the softened (or failed) volume. In the meantime, the analogy to cohesive approaches is straightforward through the definition of a damage variable. Furthermore, the implementation follows the same procedure as that of the eigenerosion algorithm except for the strength criterion for crack initiation. Consequently, the

40 current work emerges as the first alternative which combines the advantages of the three methods for the fracture quasi-brittle materials, and particularly suitable for a meshfree framework.

Moreover, the quantification of fracture energy is of great interest under dynamic loading. From the experimental part, in 1980s, using high speed photography, Mindess and Bentur [16] carried out studies of the fracture, under impact loading, for both plain and fibre reinforced concrete, as well as those with conventional reinforcement. They concluded that the cracking process under impact loading is not substantially different from that which occurs under static loading, even though the absorption of energy is quantitatively different due to the inertia effect. In a sequel, Mindess, Banthia and Yan [17] showed that both the fracture toughness and the fracture energy were dramatically increased under impact loading. Banthia et al. [18] also presented a drop-weight impact-testing machine for generating impact flexural loading; by replacing the distributed inertia load with a generalized point inertial load and subtracting it 


\section{Experimental observations}

In order to study the loading-rate effect in concrete, a drop-weight impact instrument [24], see Fig. 1, was designed and constructed in the Laboratory of Materials and Structures at the University of Castilla-La Mancha. Three-point bending tests on notched beams were conducted under impact loading. It has and can accommodate flexural specimens with spans of up to approximately $1.6 \mathrm{~m}$. In the study of Zhang et al. [22, 23], an impact hammer of $120.6 \mathrm{~kg}$ was employed to drop from three different heights, 360, 160 and $40 \mathrm{~mm}$ with the corresponding impact speeds of 2640, 1760 and $881 \mathrm{~mm} / \mathrm{s}$, respectively. The 


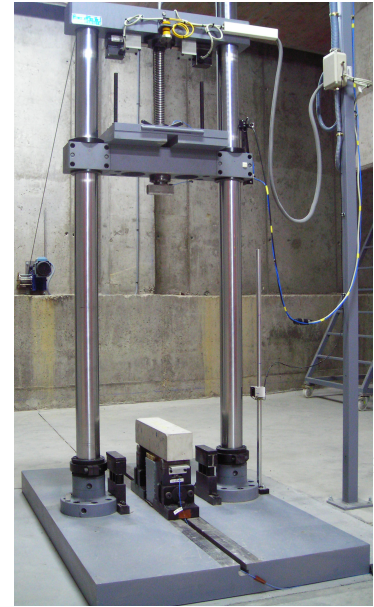

(a)

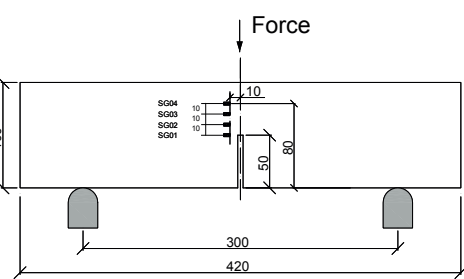

(b)

Figure 1: (a) The drop-weight machine designed at the Laboratory of Materials and Structures, University of Castilla-La Mancha [24]. (b) Geometry and dimension of the concrete beam (in $\mathrm{mm}$ ) and location of the strain gauges [23].

reaction force was determined by two force sensors located between the support and the specimen. A strain amplifier and two oscilloscopes were used to acquire the data from the strain gauges, located as it can be seen in Fig. 1.

The beam dimensions were $100 \mathrm{~mm} \times 100 \mathrm{~mm}(\mathrm{~B} \times \mathrm{D})$ in cross section, and $420 \mathrm{~mm}$ in total length (L). The initial notch-depth ratio was approximately 0.5 , and the span, S, was fixed at $300 \mathrm{~mm}$ during the tests, see Fig. 1. The specimen surface was polished and all four strain gauges were bonded to that surface, with a distance of $10 \mathrm{~mm}$ between neighboring gauges. The four strain gauges were bonded $10 \mathrm{~mm}$ apart from the centerline of the beam, see Fig. 1. Consequently, both strain history at the bonded positions, and the time at which the crack tip reaches each strain gauge [23] were obtained. 
Table 1: Mechanical properties of the high strength concrete tested by Zhang et al. [23].

\begin{tabular}{c|cccccc} 
& $\rho$ & $f_{c}$ & $f_{t}$ & $G_{F}$ & $E$ & $d_{\max }$ \\
& $\left(\mathrm{kg} / \mathrm{m}^{3}\right)$ & $(\mathrm{MPa})$ & $(\mathrm{MPa})$ & $(\mathrm{N} / \mathrm{m})$ & $(\mathrm{GPa})$ & $(\mathrm{mm})$ \\
\hline Mean & 2368 & 102.7 & 5.4 & 141 & 31 & 12 \\
Std. Dev & 1 & 2 & 0.8 & 9 & 2 & - \\
\hline
\end{tabular}

The material was characterized with independent tests and the measured material properties, such as the material density, $\rho$, compressive strength, $f_{c}$, the tensile strength, $f_{t}$, the specific fracture energy, $G_{F}$ and the elastic modulus, $E$, are given in Table 1 .

\section{The meshfree methodology}

For completeness, we first summarize the basics of the OTM scheme, the eigen-erosion algorithm to treat brittle fracture within the meshfree framework. Subsequently, the eigen-softening algorithm, particularly designed for fracture of quasi-brittle materials within the OTM framework is developed in detail.

\subsection{The OTM scheme}

OTM [14] is a meshfree updated Lagrangian numerical scheme that combines concepts from optimal transportation theory with a material-point sampling and the local max-ent meshfree approximation [26]. The method has been used in applications involving dynamic deformation and failure of materials with excellent results. The numerous advantages previously mentioned ensure its robustness and stability in numerical calculations.

Two sets of points, namely, nodal points and material points are introduced in the OTM method. A schematic of the OTM approximation scheme for an incremental motion from time $t_{k}$ to $t_{k+1}$ is shown in Figure 2. The kinematic 


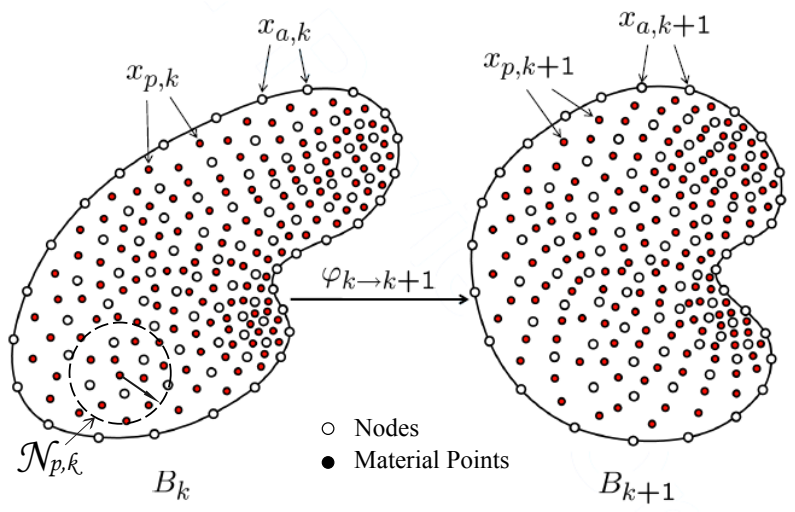

Figure 2: Schematic of the OTM approximation scheme showing the incremental motion from time $t_{k}$ to $t_{k+1}$ and the corresponding sets of nodal points (empty circles), $x_{a, k}$ and $x_{a, k+1}$, and material points (solid circles), $x_{p, k}$ and $x_{p, k+1}$, respectively.

information of the domain, such as the displacement, velocity and acceleration field, is stored at the nodes. The material responses and local state variables are evaluated at the material points, which are also transported by the incremental motion of the domain. To approximate the incremental motion from nodal displacement field, the local max-ent approximation scheme defined by Arroyo and Ortiz [26] is employed. The local max-ent function (LME), as a Pareto set, is optimal for $\beta \in(0, \infty)$ and obtained as

$$
N_{a}(\mathbf{x})=\frac{\exp \left[-\beta\left|\mathbf{x}-\mathbf{x}_{\mathbf{a}}\right|^{2}+\boldsymbol{\lambda}^{*} \cdot\left(\mathbf{x}-\mathbf{x}_{\mathbf{a}}\right)\right]}{Z\left(\mathbf{x}, \boldsymbol{\lambda}^{*}(\mathbf{x})\right)}
$$

where

$$
Z(\mathbf{x}, \boldsymbol{\lambda})=\sum_{a=1}^{n} \exp \left[-\beta\left|\mathbf{x}-\mathbf{x}_{\mathbf{a}}\right|^{2}+\boldsymbol{\lambda} \cdot\left(\mathbf{x}-\mathbf{x}_{\mathbf{a}}\right)\right]
$$

being $\boldsymbol{\lambda}^{*}(\mathbf{x})$ the unique minimizer for $\log Z(\mathbf{x}, \boldsymbol{\lambda})$. The parameter $\beta$ is related with the discretization size (or nodal spacing), $h$, whereas $\gamma$ controls the locality 
of the shape functions, as follows

$$
\beta=\frac{\gamma}{h^{2}} .
$$

For a uniform nodal spacing, $\beta$ is also a constant, thus the first derivatives can be obtained by employing the following expression

$$
\nabla N_{a}^{*}=-N_{a}^{*}\left(\mathbf{J}^{*}\right)^{-1}\left(\mathbf{x}-\mathbf{x}_{\mathbf{a}}\right),
$$

where $\mathbf{J}$ is the Hessian matrix, defined by

$$
\begin{aligned}
\mathbf{J}(\mathbf{x}, \boldsymbol{\lambda}, \beta) & =\frac{\partial \mathbf{r}}{\partial \boldsymbol{\lambda}} \\
\mathbf{r}(\mathbf{x}, \boldsymbol{\lambda}, \beta) & \equiv \partial_{\boldsymbol{\lambda}} \log Z(\mathbf{x}, \boldsymbol{\lambda}) \\
& =\sum_{a} N_{a}(\mathbf{x}, \boldsymbol{\lambda}, \beta)\left(\mathbf{x}-\mathbf{x}_{\mathbf{a}}\right) .
\end{aligned}
$$

Note that the objective of the above procedure is to find the $\boldsymbol{\lambda}$ which minimizes $\log Z(\mathbf{x}, \boldsymbol{\lambda})$. This unconstrained minimization problem with a strictly convex objective function can be solved efficiently and robustly by a combination of the Newton-Raphson method and Nelder-Mead Simplex algorithm [27, 26].

In calculations, the max-ent shape functions are reconstructed continuously from the nodal set, which changes in every step by adapting to the local deformation of the material points. This overcomes the essential difficulties of mesh entanglement that arise in Lagrangian grid-based numerical schemes. On the other hand, material points result from the spatial approximation of the mass densities $\rho_{h, k}(x)$ by $M$ points

$$
\rho_{h, k}(x)=\sum_{p=1}^{M} m_{p} \delta\left(x-x_{p, k}\right),
$$


where $x_{p, k}$ represents the position at time $t_{k}$ of material point $p, m_{p}$ is the mass of the material point and $\delta\left(x-x_{p, k}\right)$ is the Dirac-delta distribution centered at $x_{p, k}$. Material points are convected by the deformation and carry a fixed mass as well as serve the purpose of integration points for the calculation of the effective nodal forces and masses. The spatial discretization is completed by approximating the deformation mapping as

$$
\varphi_{h, k \rightarrow k+1}(x)=\sum_{a=1}^{N} x_{a, k+1} N_{a, k}(x)
$$

where $x_{a, k+1}$ is the position of node $a$ at time $t_{k+1}$, and $N_{a, k}(x)$ are max-ent shape functions defined over the configuration for node $a$ at time $t_{k}$. The interpolation at a material point $x_{p, k}$ depends solely on the nodes contained in a small local neighborhood $N_{p, k}$ as shown in Fig. 2. The reconstruction of the local neighborhoods leads to a new reconnection of the material points and the nodal set on the fly adaptively.

\subsection{Eigenerosion algorithm}

Within the context of OTM formulation, fracture can be modelled simply by failing material points according to an energy-release criterion. When the material points are failed, they are neglected from the computation of stresses in the model, which approximates the presence of cracks, this is the eigenerosion algorithm developed by Pandolfi and Ortiz [11]. Next we compute the energy-release rate attendant to the failure of material point $p$ as is it seen in $[10,11,15,12]$.

$$
\begin{aligned}
G_{p, k+1} & =\frac{C \epsilon}{m_{p, k+1}} \sum_{x_{q, k+1} \in B_{\epsilon}\left(x_{p, k+1}\right)} m_{q} W_{k}\left(F_{q, k+1}\right), \\
m_{p, k+1} & =\sum_{x_{q, k+1} \in B_{\epsilon}\left(x_{p, k+1}\right)} m_{q},
\end{aligned}
$$




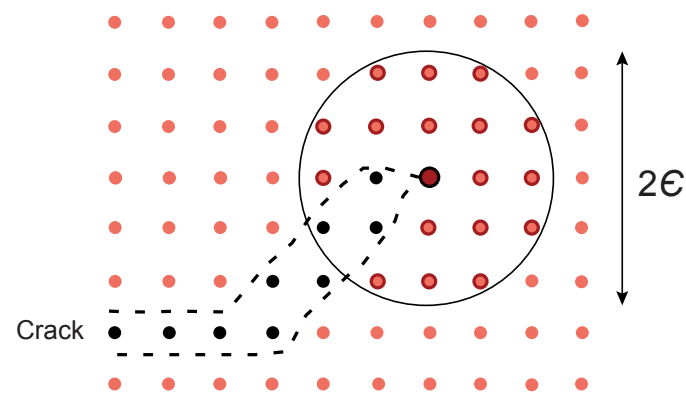

Figure 3: Scheme of a crack (black dots) as set of failed material points, and of the $\epsilon$ neighborhood (inside the circle) of the material point located at the crack tip (red dots).

where $B_{\epsilon}\left(x_{p, k+1}\right)$ is the sphere of radius $\epsilon$ centered at $x_{p, k+1}$ known as the $\epsilon$ neighborhood of the material point, $m_{p, k+1}$ is the mass of the neighborhood at loading step $k+1, W_{k}\left(F_{q, k+1}\right)$ is the current free-energy density per unit mass at the material point $x_{q, k+1}$ and $C$ is a normalizing constant. A scheme of the configuration of the $\epsilon$-neighborhood is plotted in Fig. 3 .

The material point is failed when $G_{p, k+1}$ surpasses a critical energy release rate that measures the material-specific energy, $G_{F}$, required to create a fracture surface of unit area. In calculations, the failed material-point set is updated at every time step according to this criterion. For linear elasticity, Schmidt et $a l$. [10] have shown that this approximation converges to Griffith fracture in the limit of an infinitely fine discretization. In order to minimize the computational effort, employing an averaging over an intermediate $\epsilon$-neighborhood to compute the energy-release rate attendant to material-point failure, Eq. (9), is essential to convergence. Indeed, erosion schemes that estimate the energy-release rate based on the energy of a single material point suffer from mesh-dependency and may overestimate the toughness of the material. It needs to be pointed out that when a material point satisfies the erosion condition, its contribution to the internal force vector and to the material stiffness matrix is set to zero, but its contribution to the mass matrix is maintained. The mass of a material point 
175 is discarded only when an eroded material point is not connected to any nodes.

\subsection{Eigensoftening algorithm}

From different numerical simulations carried out using the eigenerosion algorithm for modeling fracture in concrete (see next Section), the main drawback fact leads to an unrealistic stress concentration, reaching values significantly higher than the tensile strength of quasi-brittle materials. This is attributed to the fact that an energetic criterion is adopted in the eigenerosion algorithm. In order for a material point to be eroded, the strain energy accumulated must reach the given value, $G_{F}$. Such an energy accumulation is necessarily obtained through higher values of stresses (and strains) in quasi-brittle materials. In the limit case, infinitely high stresses will be reached, such is the case of infinite stress concentration at the crack tip in Linear Elastic Fracture Mechanics. To overcome this limitation, we propose the concept of eigensoftening to take into account the gradual failure in quasi-brittle materials. This is similar to that of cohesive fracture. For instance, in Fig. 4, a linear decreasing cohesive law is plotted, where the shaded area represents the static fracture energy per unit of area, $G_{F}$. When the maximum tensile strength, $f_{t}$, is reached, a cohesive crack is formed with zero opening displacement. Once the opening displacement, $w$, reaches a critical vale, $w_{c}$, a stress-free crack is attained. For intermediate values, $w_{n}$, a damage value between zero and one represents the extension to which the material has failed.

The implementation of the eigensoftening algorithm consists in adopting a strength criterion for crack initiation and a softening law which is proper to the material under study before the formation of a stress-free crack. Following the philosophy of eigenerosion, we rewrite Eq. (9) in terms of the principal stresses 


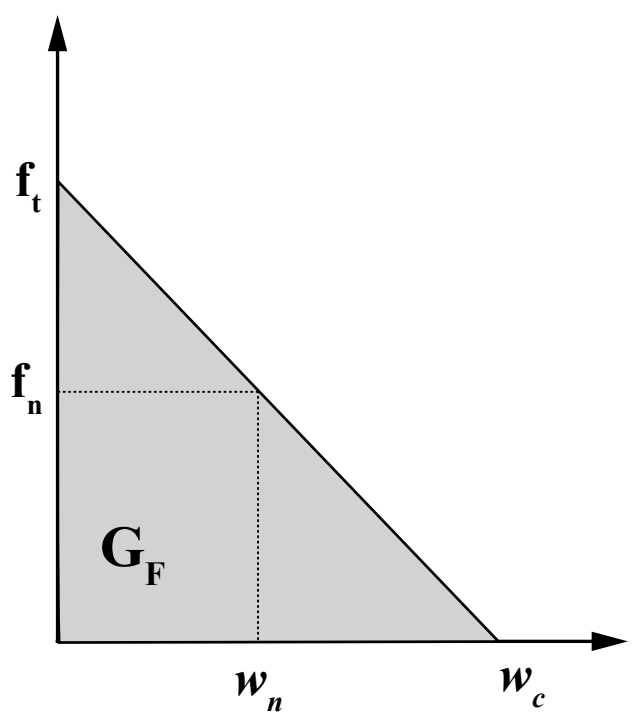

Figure 4: Scheme of a linear cohesive law, where the shade area is $G_{F}, f_{t}$ is the tensile strength, and $w_{c}$ is the critical opening displacement.

at time $t_{k+1}$. In specific, we consider the maximum principal stress theory for brittle fracture. To this end, the variation of the averaged strain energy density in the $\epsilon$-neighborhood of the material point $x_{p, k+1}$ can be expressed as,

$$
\delta W_{p}^{\epsilon}=\frac{\partial G_{p}}{C \epsilon}=\frac{1}{m_{p}} \sum_{x_{q} \in B_{\epsilon}\left(x_{p}\right)} m_{q} \sigma_{q, 1} \delta \varepsilon_{q}
$$

where $\sigma_{q, 1}$ is the maximum principal stress at a neighboring material point $x_{q, k+1}$ for a general three dimensional problem. We introduce here an effective strain $\varepsilon_{q}$ at the material point $x_{q, k+1}$, such that the variation of the local strain energy can be obtained as $\delta W_{q}=\sigma_{q, 1} \delta \varepsilon_{q}$. In addition, assume the effective strain increment of each material point at every time step can be approximated by its counterpart in the neighborhood. This is due to the fact that there is no significant variation when the space discretization is small enough. Conse- 
quently, Eq. (10) is simplified as follows,

$$
\delta W_{p}^{\epsilon}=\frac{\delta \varepsilon_{p}}{m_{p}} \sum_{x_{q, k+1} \in B_{\epsilon}\left(x_{p, k+1}\right)} m_{q} \sigma_{q, 1} .
$$

With the definition of an equivalent critical stress at the material point $x_{p, k+1}$ :

$$
\begin{aligned}
\sigma_{p}^{\epsilon} & =\frac{1}{m_{p}} \sum_{x_{q, k+1} \in B_{\epsilon}\left(x_{p, k+1}\right)} m_{q} \sigma_{q, 1} \\
\text { where } \quad m_{p} & =\sum_{x_{q, k+1} \in B_{\epsilon}\left(x_{p, k+1}\right)} m_{q},
\end{aligned}
$$

we obtain the conjugate relationship between the equivalent critical stress and the averaged strain energy density, i.e., $\delta W_{p}^{\epsilon}=\sigma_{p}^{\epsilon} \delta \varepsilon_{p}$. Once the first principal stress, the maximum eigenvalue of the Cauchy stress tensor, is determined for every intact material point, its average over an $\epsilon$-neighborhood defined in Eq. (12) can be easily calculated. When $\sigma_{p, k+1}^{\epsilon}$ surpasses the tensile strength, $f_{t}$, the softening behavior is activated, which in turn causes a reduction of the 220 internal forces as follows,

$$
f^{i n t}=(1-\chi) \sum^{p} \int_{\Omega} \sigma_{p, k+1} \nabla N d V
$$

where $\chi$ is the damage variable, ranges between zero (an intact material) and one (completely failed material points). For the case of a linear softening behavior described in Fig. 4, it is calculated as,

$$
1-\chi=\frac{f_{n}}{f_{t}}=1-\frac{w_{n}}{w_{c}} \quad \longrightarrow \quad \chi=\frac{w_{n}}{w_{c}}
$$

Since the energy dissipation is within a volume rather than a surface as the 225 case of cohesive fracture, a band with, $h_{\epsilon}$, equivalent to the crack band model of Bažant [6] is introduced. The effective fracture strain, $\varepsilon_{f}^{\epsilon}$, defined as the 
difference between the strain at crack initiation, $\varepsilon_{1}\left(x_{p, 0}\right)$, and the current strain, $\varepsilon_{1}\left(x_{p, k+1}\right)$, for material point $p$. At the same time, $\varepsilon_{f}^{\epsilon}$ can also be represented as the current crack opening $w_{n}$ within the band width, $h_{\epsilon}$. Consequently,

$$
\varepsilon_{f}^{\epsilon}=\varepsilon_{1}\left(x_{p, k+1}\right)-\varepsilon_{1}\left(x_{p, 0}\right)=\frac{w_{n}}{h^{\epsilon}}
$$

Combing Eq. (14) and Eq. (15), the damage variable can be computed as

$$
\chi=\frac{\varepsilon_{f}^{\epsilon} h^{\epsilon}}{w_{c}}
$$

for a linear softening behavior. For a generic softening behavior, the damage variable can be expressed in terms of the following variables,

$$
\chi=\chi\left(\varepsilon_{f}^{\epsilon}, h^{\epsilon}, f_{t}, w_{c}, G_{f}\right) .
$$

It bears emphasis that a reference value for $h^{\epsilon}$ is between two and four times the maximum size of the aggregates for concrete according to Bažant [28].

\section{Numerical results and discussion}

In this Section, we compare the results obtained with the aforementioned methodologies on the simulation of the dynamic fracture propagation in a threepoint-bend beam impacted by a drop-weight device in a $2 \mathrm{D}$ setting. Both the projectile (the hammer) and the target (the concrete beam) are explicitly represented. Several levels of discretization are employed to assess the objectiveness of the obtained results. The results obtained in this Section are from a discretization of 3980 nodes, 7534 material points, and a nodal spacing of $0.65 \mathrm{~mm}$ near the middle section is maintained.

First we validate the reaction and impact forces as well as the load-line 

sured from the strain gauges with the numerical results obtained from both algorithms, see Fig. 7. Note that the results from the eigensoftening algorithm reproduce well both the general trend and the peak strains measured from the strain gauges, whereas those from the eigenerosion algorithm overestimate the

displacement against their experimental counterparts, the strain evolutions are compared with those measured from strain gauges. Next the horizontal stress distributions, crack front evolutions and crack propagation velocities are extracted. Finally the energy evolution is assessed. All the results are presented for the three different experimental loading velocities.

\subsection{Validation against experimental results}

Since the impact forces applied by the hammer and the reaction forces at the two supports were experimentally measured, they are compared with the numerical ones in Fig. 5 for the different impact velocities. Note that the general trend of both forces are correctly captured with both methods. However, lower reaction values are observed in the eigensoftening results compared to those of eigenerosion, since the fracture occurs earlier. Additionally plotted in Fig. 5 are the recorded impact-line displacements contrasted with the numerical ones. The displacement of the hammer fits quite well with the experimental measurement. Nevertheless, a discrepancy in displacements between the hammer and the top centerline of the beam is observed when eigensoftening is employed. This indicates a separation between the projectile and the beam during the impact process, observable from high-speed videos, is correctly captured. It is another evidence that the eigensoftening algorithm is suitable for modeling the fracture of quasi-brittle materials.

The second validation is carried out by comparing the strain history meapeak strain values by a factor of close to ten. 

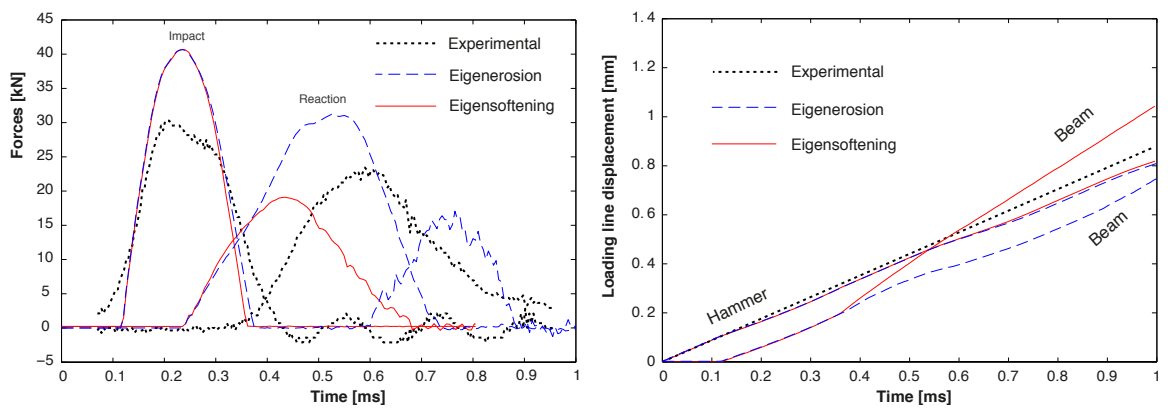

(a)
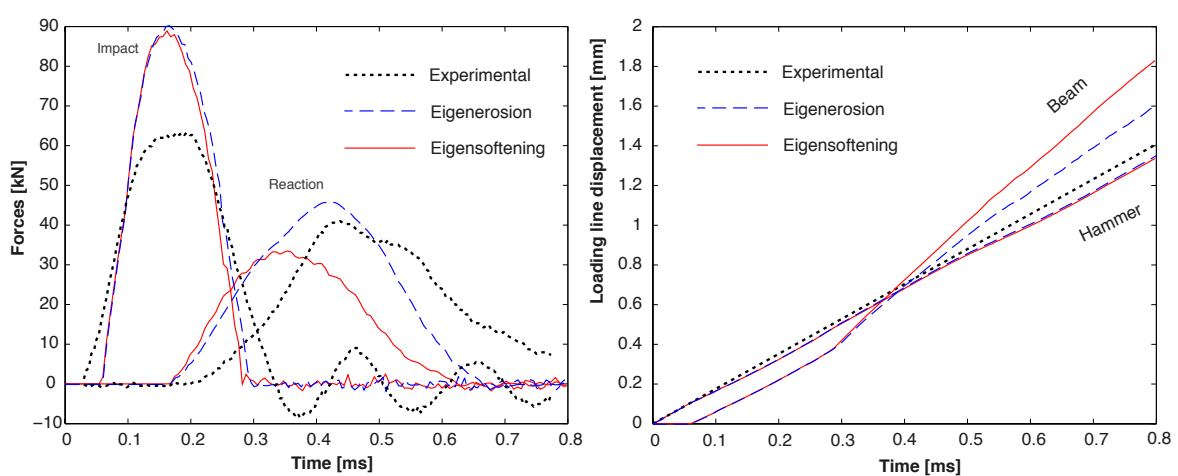

(b)
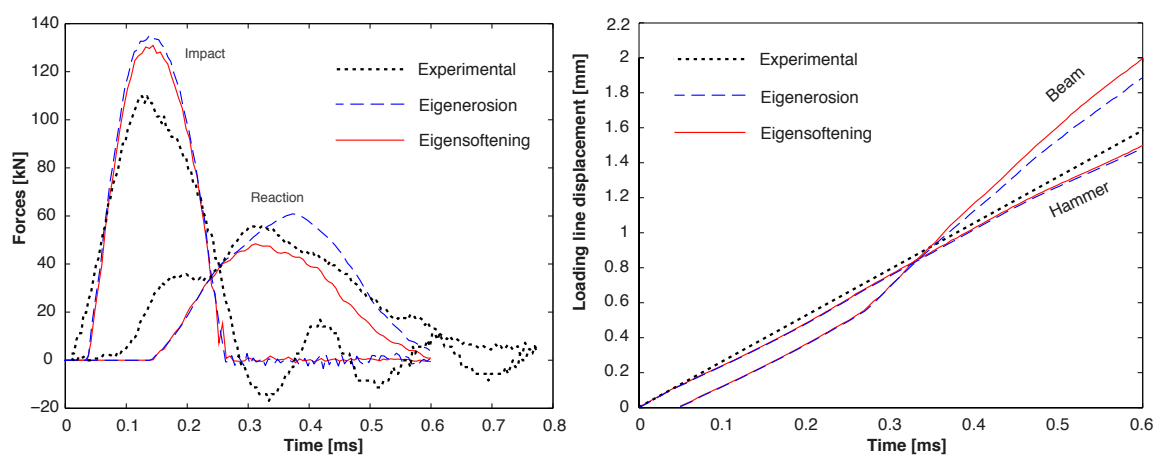

(c)

Figure 5: Comparison between experimental and computational impact and reaction forces (left) and impact-line displacements (right) for (a) $881 \mathrm{~mm} / \mathrm{s}$; (b) $1760 \mathrm{~mm} / \mathrm{s}$; and (c) $2640 \mathrm{~mm} / \mathrm{s}$. 

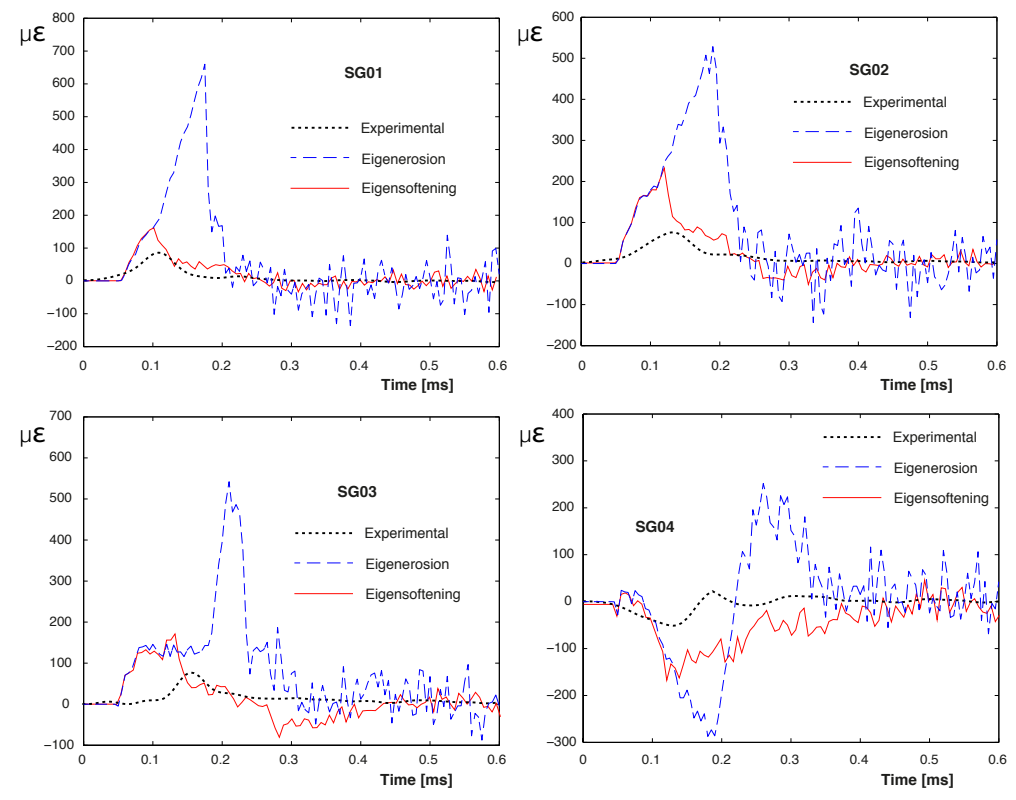

(a)
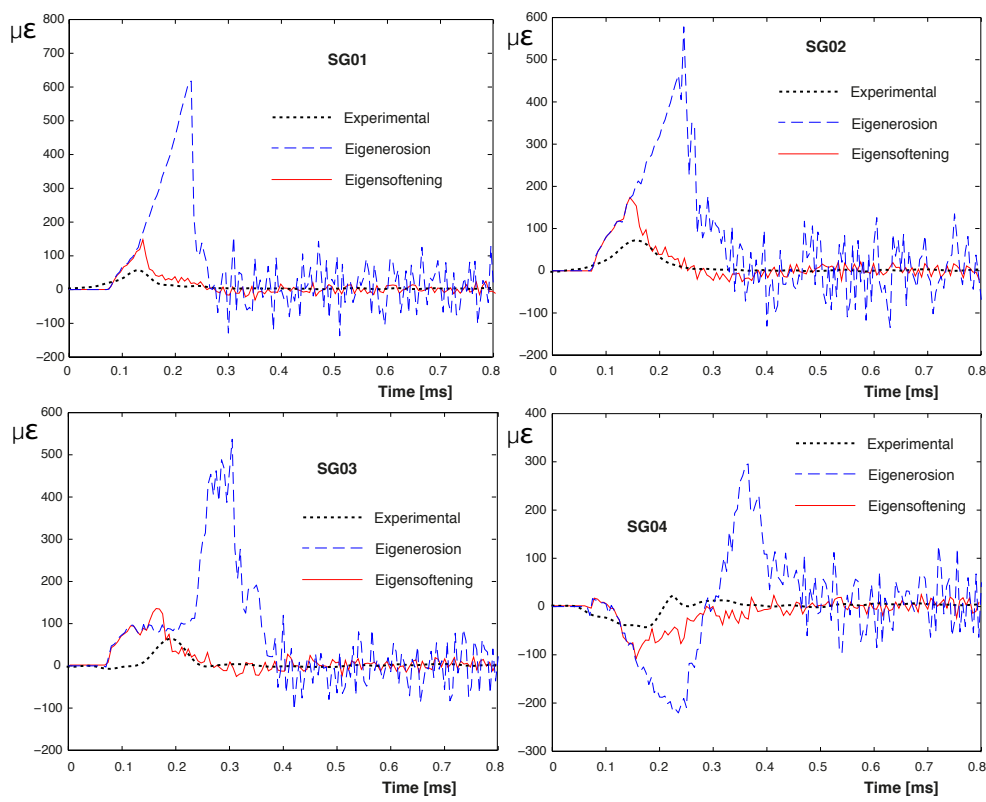

(b) 

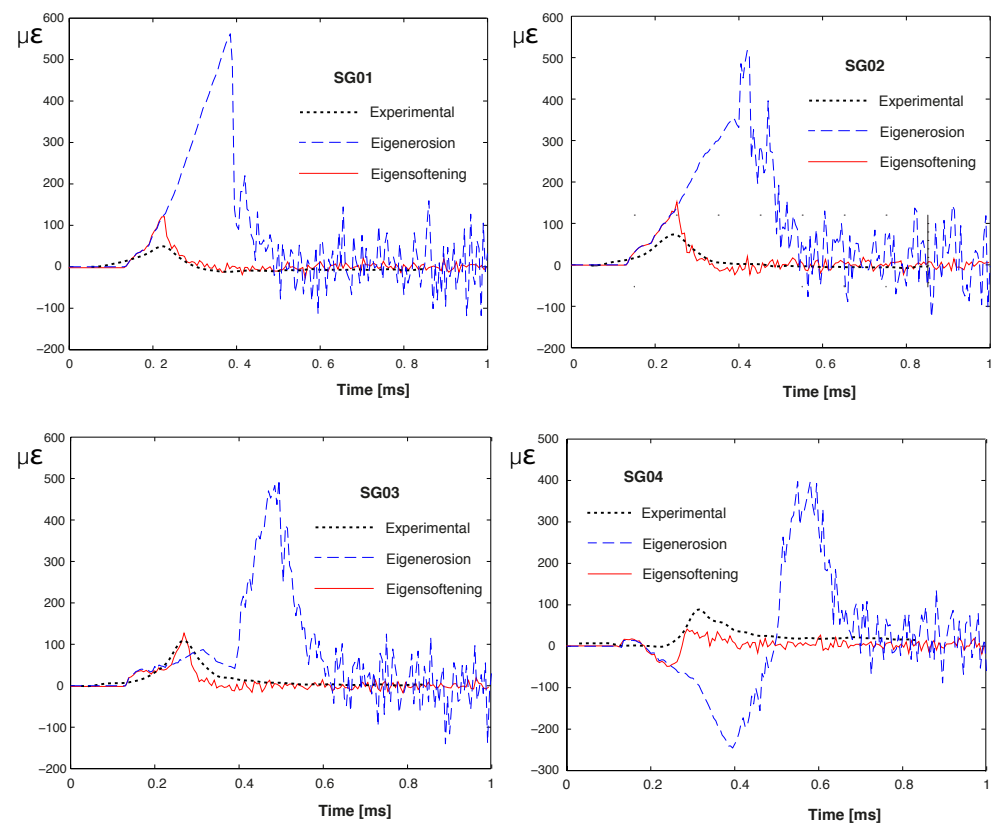

(c)

Figure 7: Numerically obtained strain evolution at the same locations where the four strain gauges are bonded and the crack-front position with respect to time for (a) $2640 \mathrm{~mm} / \mathrm{s}$; (b) $1760 \mathrm{~mm} / \mathrm{s}$; and (c) $881 \mathrm{~mm} / \mathrm{s}$. 


\subsection{Stress evolution and dynamic increase factor}

In order to look at the dynamic effect on the stress amplitudes, we illustrate the stress distributions (in horizontal direction) at three time shots for all three impact velocities in Fig. 9. Note that even though the plotted stress range 275 is limited between -10 and $10 \mathrm{MPa}$, significant higher values are observed for those results from the eigenerosion algorithm. Consequently, larger areas of stress concentration near the crack tip are perceived. Indeed, the dynamic increase factors (DIF) of the tensile stresses are 7.0, 8.2 and 8.5 for loading velocity at $881 \mathrm{~mm} / \mathrm{s} 1760 \mathrm{~mm} / \mathrm{s}$ and $2640 \mathrm{~mm} / \mathrm{s}$, respectively, see Fig. 10. By contrast, when eigensoftening is employed, the corresponding DIF for the tensile stresses reached only 1.14, 1.24 and 2.13 (see Table 2) for impact velocities of $881 \mathrm{~mm} / \mathrm{s} 1760 \mathrm{~mm} / \mathrm{s}$ and $2640 \mathrm{~mm} / \mathrm{s}$, respectively. These results together with the absolute values of the tensile stresses are also plotted in Fig. 10. It needs to be pointed out that, a DIF for tensile strength up to 1.8 was obtained by Beda 285 et al. [29].

It is also worth noting that in Fig. 9 crack branches are formed for two higher impact velocities. This indicates that the excess energy supply is dissipated through creating more micro or macro cracks. Similar results were obtained on dynamic fracture propagation in concrete with rebars [30, 31].

Table 2: Dynamic Increase Factor (DIF) for tensile strength and fracture energy obtained from the eigensoftening calculations.

\begin{tabular}{c|cc|cc}
$\begin{array}{c}\text { Impact Velo. } \\
{[\mathrm{mm} / \mathrm{s}]}\end{array}$ & $\begin{array}{c}\sigma_{\max } \\
{[\mathrm{MPa}]}\end{array}$ & $\begin{array}{c}\text { DIF } \\
\text { Diss. Energy } \\
{[\mathrm{J}]}\end{array}$ & $\begin{array}{c}\text { DIF } \\
-\end{array}$ \\
\hline 881 & 5.8 & 1.14 & 0.75 & 1.06 \\
1760 & 6.3 & 1.24 & 1.00 & 1.41 \\
2640 & 10.9 & 2.13 & 1.50 & 2.12 \\
\hline
\end{tabular}

In order to gain more insights into the crack propagation velocities, the 


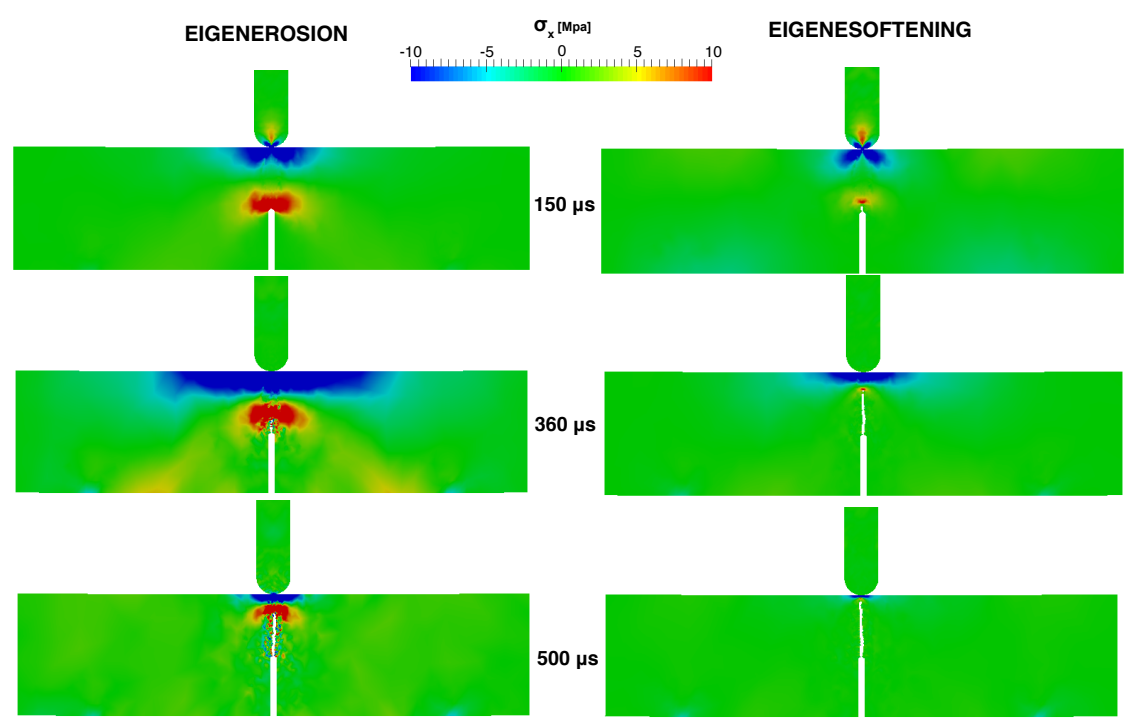

(a)

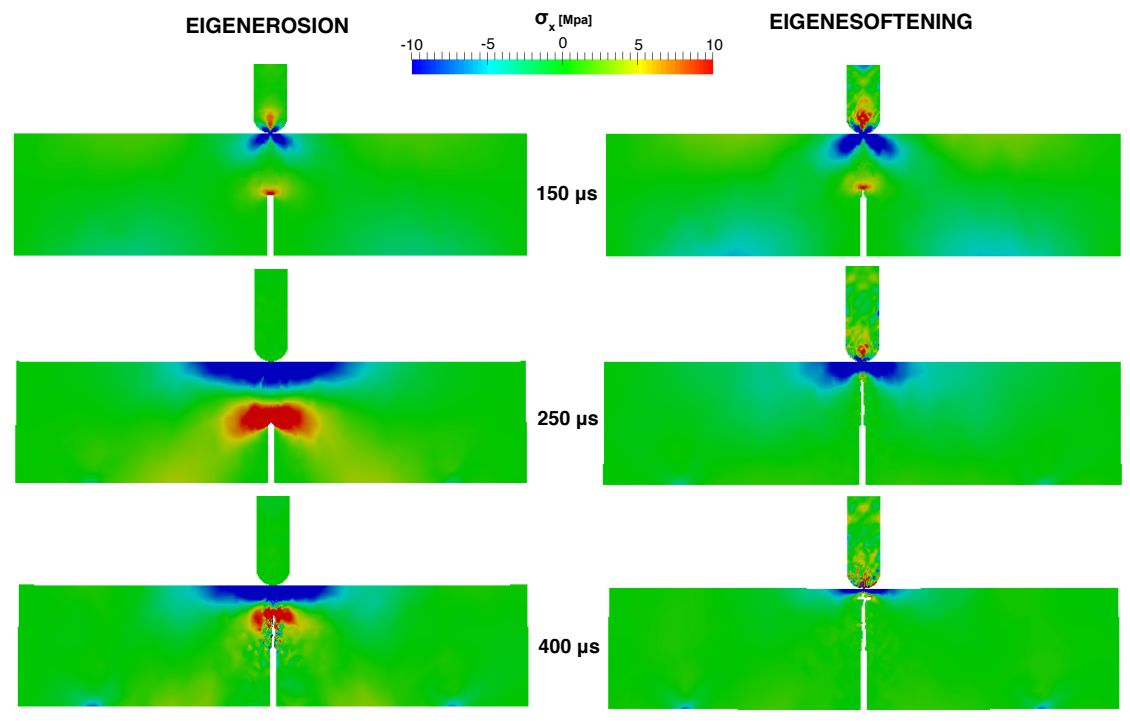

(b) 


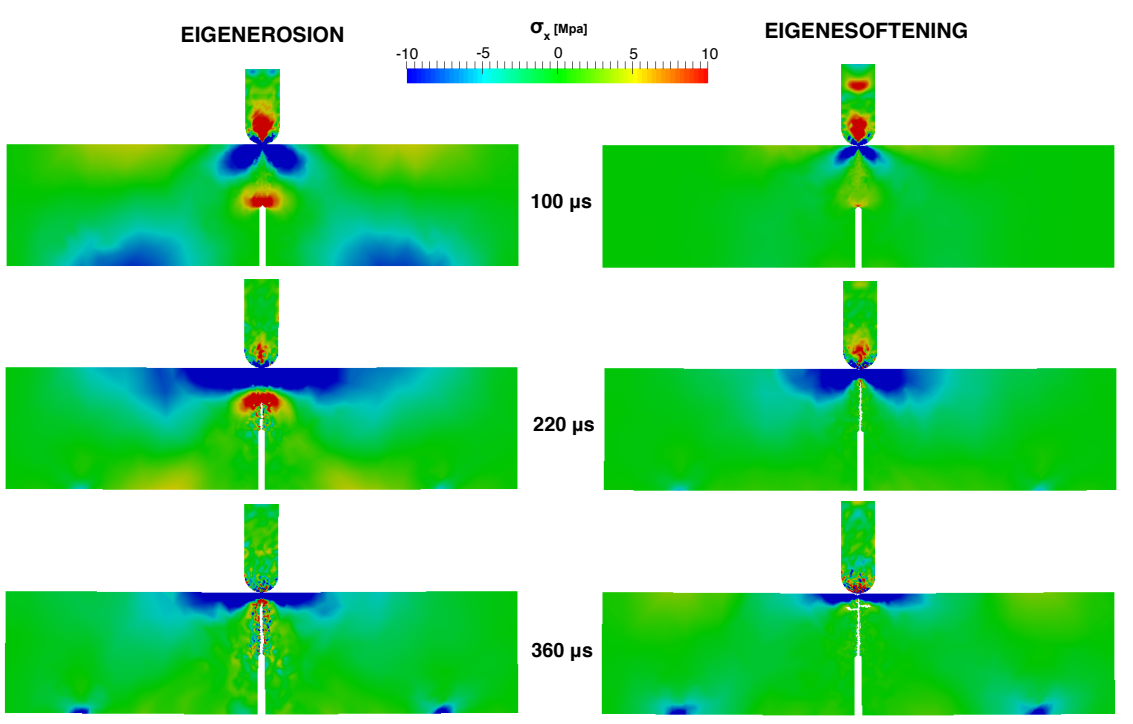

(c)

Figure 9: Evolution of the stresses for eigenerosion and eigensoftening approaches for (a) 881 $\mathrm{mm} / \mathrm{s}$; (b) $1760 \mathrm{~mm} / \mathrm{s}$; and (c) $2640 \mathrm{~mm} / \mathrm{s}$.

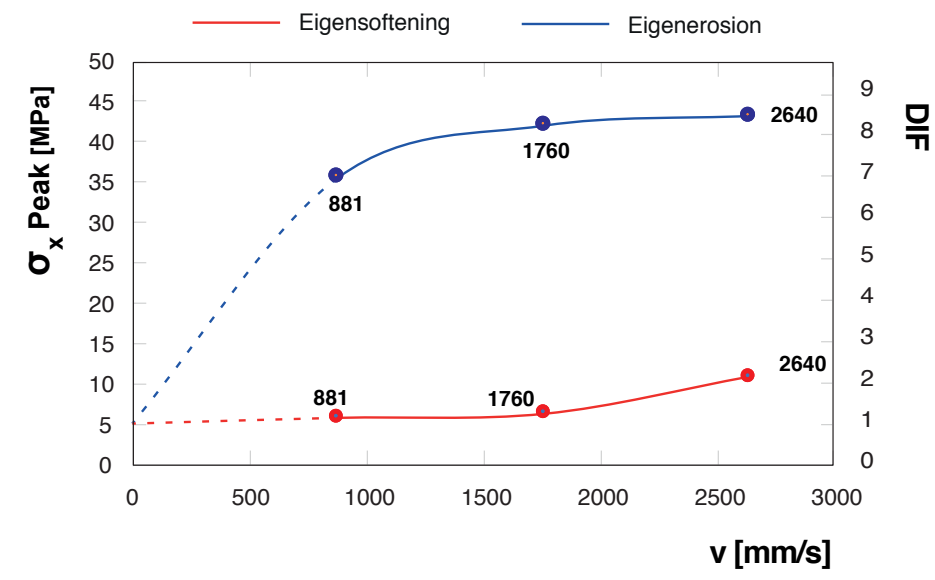

Figure 10: Peak values of the tensile stresses and the corresponding DIFs for different loading velocities calculated with eigenerosion and eigensoftening models. 
highest most recently eroded material points are extracted as crack fronts and plotted with respect to time in Fig. 11. Subsequently, derivatives are taken to obtain the crack propagation velocities. Note that the peak values of the crack velocity and the decreasing trend agree very well with the experimentally measured ones given by Zhang et al. [23] for both methods. Nevertheless, the crack speed obtained from eigensoftening are faster since it facilitates the crack initiation and propagation. 

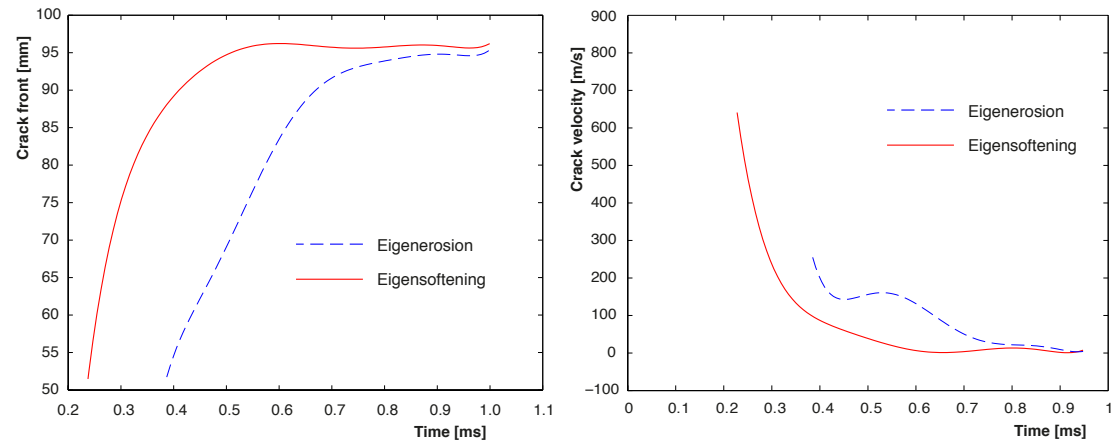

(a)
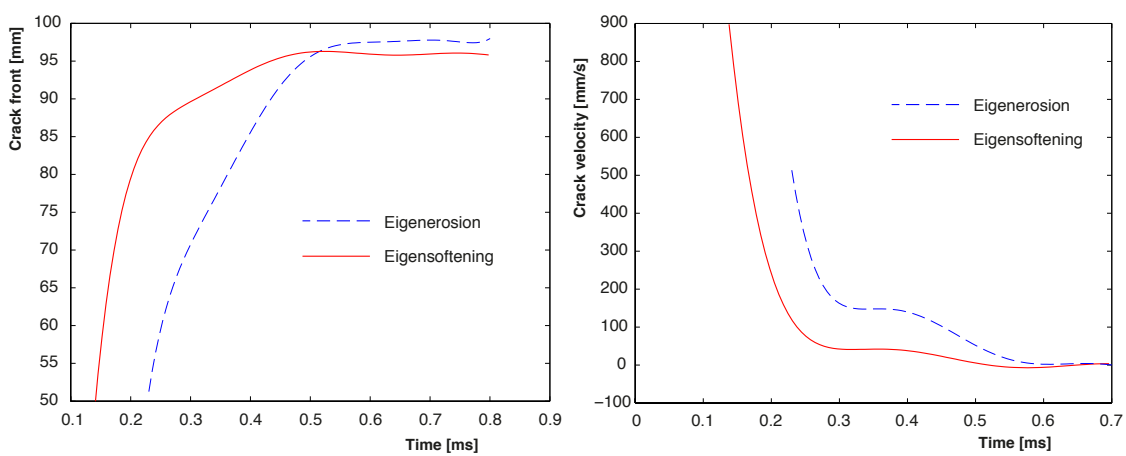

(b)
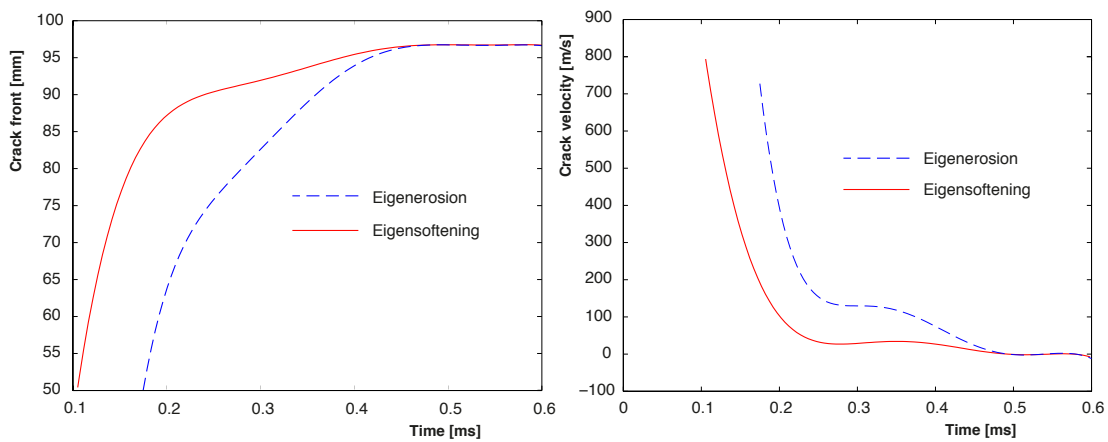

(c)

Figure 11: Crack front position (left column) and crack front velocity (right column) for both eigenerosion and eigensoftening models for (a) $881 \mathrm{~mm} / \mathrm{s}$; (b) $1760 \mathrm{~mm} / \mathrm{s}$; and (c) $2640 \mathrm{~mm} / \mathrm{s}$. 


\subsection{Energy evolution}

Another interesting phenomenon is the quantification of fracture energy ex-

$$
\bar{\chi}=\frac{\sum \chi A_{d}}{\sum A_{d}} .
$$

The three values for the three impact velocities are listed in Tab. 3. As expected, the total damaged area (with or without the weights of $\chi$ ) grow with the impact 


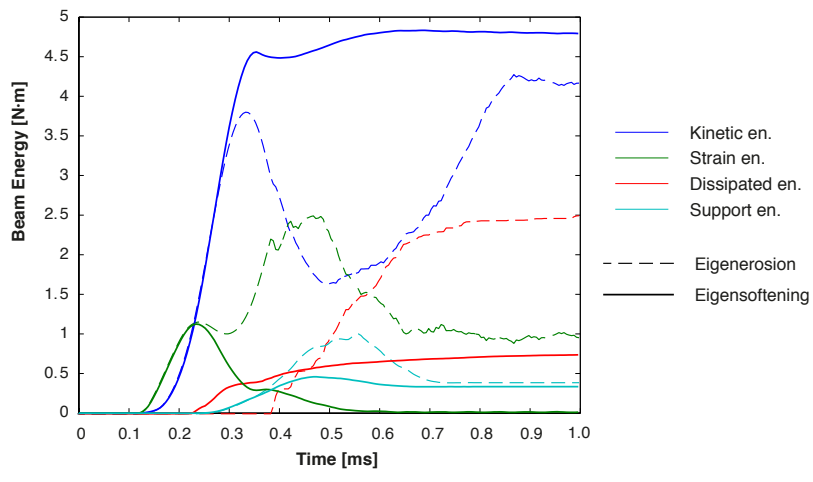

(a)

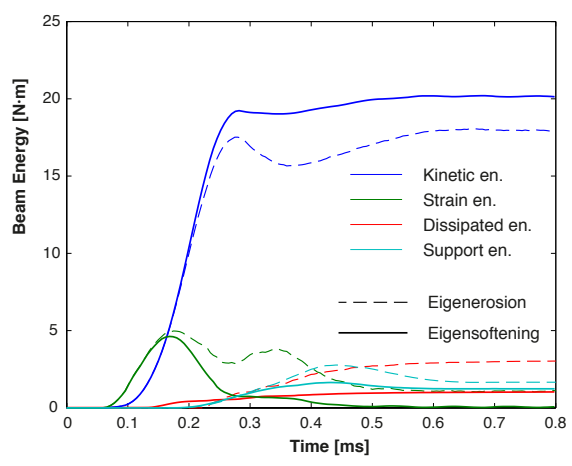

(b)

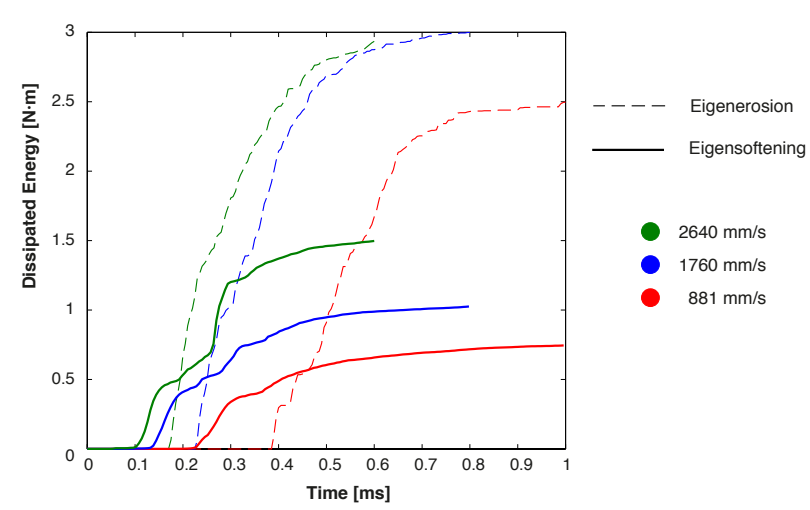

(d)

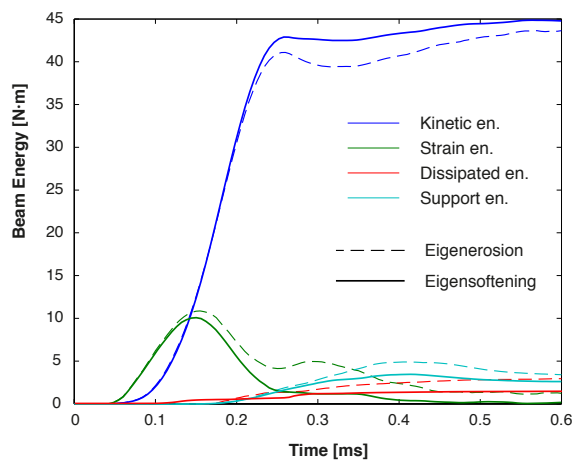

(c)

Figure 12: Numerical energy evolution for both eigenerosion and eigensoftening methodologies for (a) $881 \mathrm{~mm} / \mathrm{s}$; (b) $1760 \mathrm{~mm} / \mathrm{s}$; and (c) $2640 \mathrm{~mm} / \mathrm{s}$; and (d) the zoomed view of the fracture energy expenditure. 


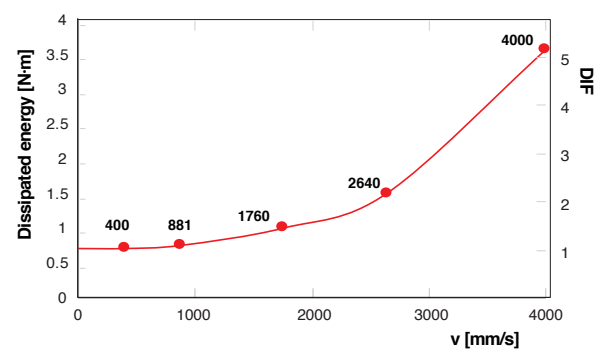

Figure 13: Fracture energy dissipation and the corresponding DIF for impact velocities at $400,881,1760,2640$ and $4000 \mathrm{~mm} / \mathrm{s}$.

velocity. Nevertheless, the average damage is independent of the loading rate.

325 This indicates that the DIF in fracture energy is the result of failing more material points rather than more severely failing the same material. Similar conclusions were arrived in the work of Ruiz et al. [32] for the modelling of the failure of concrete cylinders through cohesive models.

Table 3: Total damaged area, average damage and the total damaged area weighted with its $\chi$ values.

\begin{tabular}{c|ccc} 
& $\sum A_{d}\left[\mathrm{~mm}^{2}\right]$ & $\bar{\chi}$ & $\sum \chi A_{d}\left[\mathrm{~mm}^{2}\right]$ \\
\hline $2640 \mathrm{~mm} / \mathrm{s}$ & 1385.6 & 0.222 & 307.4 \\
$1760 \mathrm{~mm} / \mathrm{s}$ & 822.7 & 0.290 & 240.4 \\
$881 \mathrm{~mm} / \mathrm{s}$ & 599.1 & 0.271 & 162.4 \\
\hline
\end{tabular}




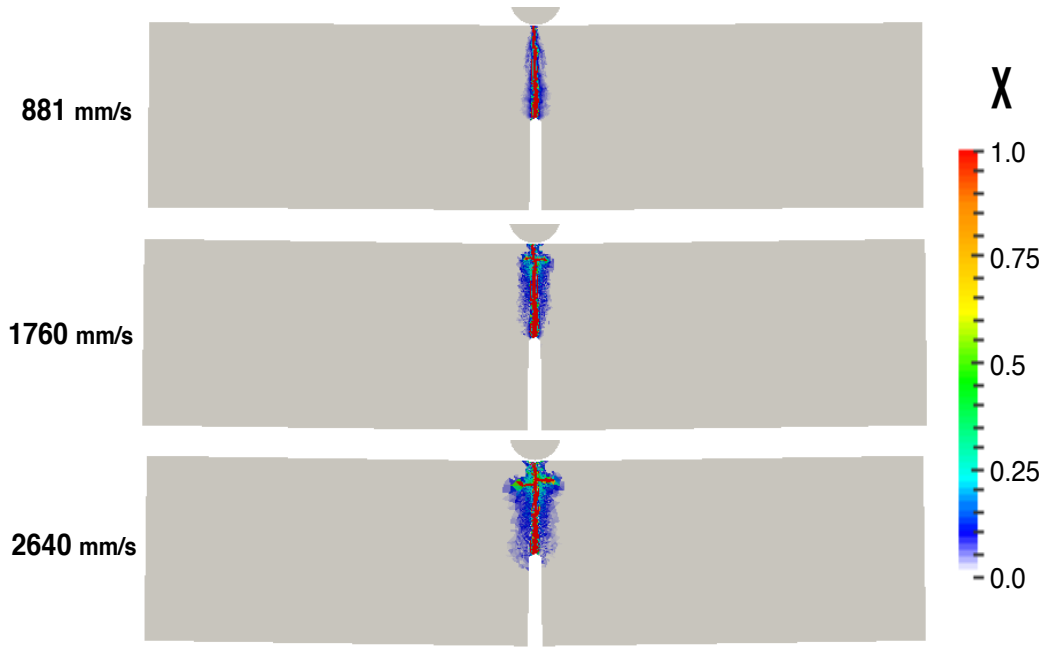

Figure 14: Damage levels at the end of calculations for impact velocities at 881,1760 and $2640 \mathrm{~mm} / \mathrm{s}$. 


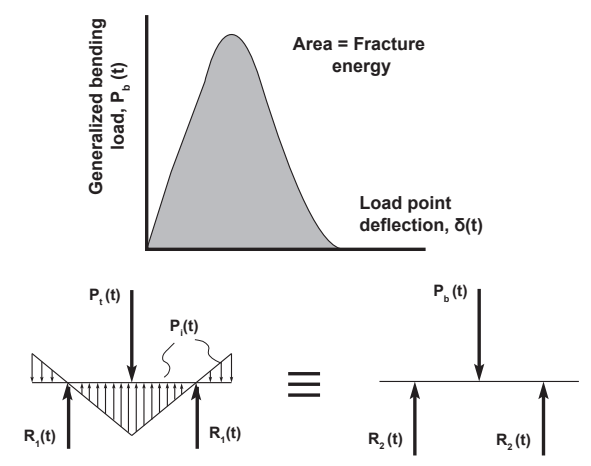

Figure 15: Use the equivalent reaction (bending load) to calculate the fracture energy under dynamic loading (via a drop-weigh device).

\subsection{Influence on the measurement of dynamic fracture energy}

In order to experimentally measure the dynamic fracture energy, it is essential to take off the influence of inertia forces. Banthia et al. [18] proposed to employ the area below the reaction-deflection curve $(P-\delta)$, see Fig. 15. In this Section, we endeavor to assess such a procedure through the results from the aforementioned methodology with eigenerosion algorithm.

In Fig. 16, we plot the total energy of beam with (green solid lines) and without (red discontinuous lines) the dissipated energy contrasted with the $P-\delta$ integral (blue dotted lines). Note that there is insignificant difference between the green and blue curves for all five impact velocities considered. However, the difference between the red and green curves decreases with the impact velocity. In other words, the percentage of the dissipated fracture energy with respect to the total energy inside the beam decreases significantly with the increase of the impact velocity. This is clearly shown in Fig. 17. It is noteworthy that the area below the $P-\delta$ curve may indeed approaches the dissipated energy at static cases, in the dynamic case, however, it closely matches the total energy of the beam, whereas the fracture energy is solely a small percentage (3\% when the impact velocity is higher than $2640 \mathrm{~mm} / \mathrm{s}$ ). 

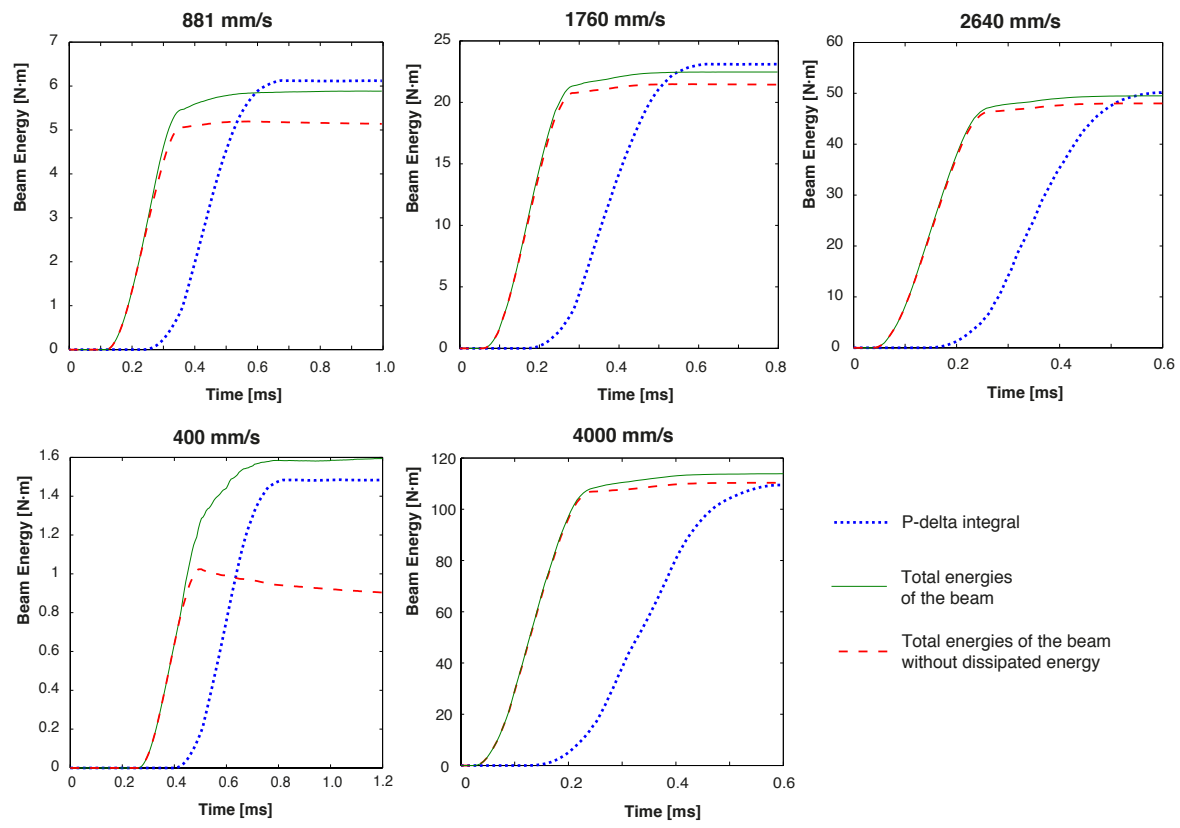

Figure 16: Comparison of the beam energy with (green) or without (red) without the dissipated fracture energy with the integral of the P-delta curve (blue) for different impact velocities.

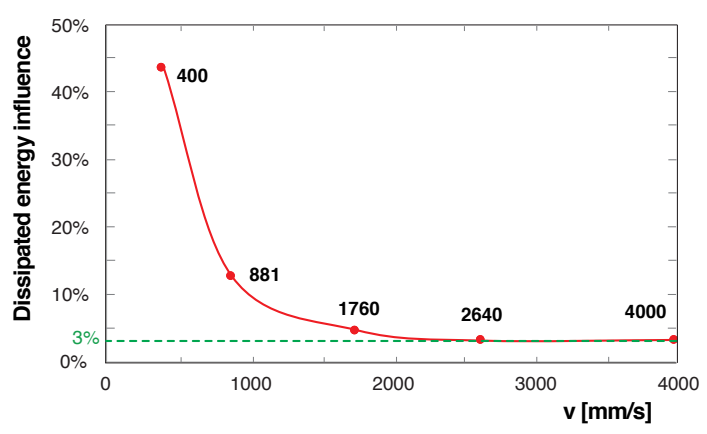

Figure 17: Variation of the percentage of the dissipated fracture energy with respect to the total energy in the beam with the loading velocities. 


\section{Summary and conclusions}

Based on the eigenerosion approach to fracture, we have developed an eigensotening algorithm for the fracture of quasi-brittle materials. The former con-

350 


\section{Acknowledgements}

We acknowledge the financial support from the Ministerio de Economia y Competitividad through grant number BIA2015-68678-C2-R and from Conse-

375 jería de Educación, Cultura y Deportes, Junta de Comunidades de Castilla-La Mancha, Spain \& Fondo Europeo de Desarrollo Regional (FEDER) through grant number PEII-2014-016-P.

\section{References}

[1] G. Barenblatt, The mathematical theory of equilibrium cracks in brittle fracture, Advances in Applied Mechanics 7 (1962) 55-129.

[2] A. Hillerborg, M. Modéer, P. Petersson, Analysis of crack formation and crack growth in concrete by means of fracture mechanics and finite elements, Cement and Concrete Research 6 (1976) 773-782.

[3] M. Ortiz, A. Pandolfi, Finite-deformation irreversible cohesive elements for three-dimensional crack-propagation analysis, International Journal for Numerical Methods in Engineering 44 (1999) 1267-1282.

[4] A. Pandolfi, M. Ortiz, An efficient adaptive procedure for three-dimensional fragmentation simulations, Engineering with Computers 18(2) (2002) $148-159$.

[5] G. Ruiz, A. Pandolfi, M. Ortiz., Three-dimensional cohesive modeling of dynamic mixed-mode fracture, International Journal for Numerical Methods in Engineering 52 (2001) 97-120.

[6] Z. Bažant, B. Oh, Crack band theory for fracture in concrete, Materials and Structures 16 (1983) 155-177. 

on loss of hyperbolicity and a new discontinuous enrichment, International Journal for Numerical Methods in Engineering 58 (2003) 1873-1905.

[8] A. Gravouil, N. Moës, T. Belytschko, Non-planar 3D crack growth by the extended finite element and level sets-Part II: level set update, International Journal for Numerical Methods in Engineering 53 (2002) 2569-2586.

[9] T. Rabczuk, T. Belytschko, Cracking particles: a simplified meshfree method for arbitrary evolving cracks., International Journal for Numerical Methods in Engineering 61 (2004) 2316-2343.

[10] B. Schmidt, F. Fraternali, M. Ortiz, Eigenfracture: an eigendeformation approach to variational fracture, SIAM J. Multiscale Model. Simul. 7 (2009) $1237-1266$.

[11] A. Pandolfi, M. Ortiz, An eigenerosion approach to brittle fracture, International Journal for Numerical Methods in Engineering 92 (2012) 694-714.

[12] A. Pandolfi, B. Li, M. Ortiz, Modeling fracture by material-point erosion, International Journal of fracture 184 (2013) 3-16.

[13] B. Li, A. Pandolfi, M. Ortiz, Material-point erosion simulation of dynamic fragmentation of metals, Mechanics of Materials 80 (2015) 288-297.

[14] B. Li, F. Habbal, M. Ortiz, Optimal transportation meshfree approximation schemes for fluid and plastic flows, International Journal for Numerical Methods in Engineering 83 (2010) 1541-1579.

[15] B. Li, A. Kadane, G. Ravichandran, M. Ortiz, Verification and validation of the optimal-transportation meshfree (OTM) simulation of terminal ballistics, International Journal for Impact Engineering 42 (2012) 25-36. 
[16] S. Mindess, A. Bentur, A preliminary study of the fracture of concrete beams under impact loading, using high-speed photography, Cement and Concrete Research 15(3) (1985) 474-484.

[17] S. Mindess, N. Banthia, C. Yan, The fracture-toughness of concrete under impact loading, Cement and Concrete Research 17(2) (1986) 231-241.

[18] N. Banthia, S. Mindess, A. Bentur, M. Pigeon, Impact testing of concrete using a drop-weight impact machine, Experimental Mechanics 29(1) (1989) 63-69.

[19] N. Banthia, S. Mindess, J. Trottier, Impact resistance of steel fiber reinforced concrete, ACI Materials Journal 93(5) (1996) 472-479.

[20] N. Wang, S. Mindess, K. Ko, Fiber reinforced concrete beams under impact loading, Cement and Concrete Research 26(3) (1996) 363-376.

[21] N. Banthia, C. Yan, K. Sakai, Impact resistance of concrete plates reinforced with a fiber reinforced plastic grid, ACI Materials Journal 95(1) (1998) 11-18.

[22] X. Zhang, G. Ruiz, R. Yu, M. Tarifa, Fracture behaviour of high-strength concrete at a wide range of loading rates, International Journal of Impact Engineering 36 (2009) 1204-1209.

[23] X. Zhang, R. Yu, G. Ruiz, M. Tarifa, M. Camara, Effect of loading rate on crack velocities in HSC, International Journal of Impact Engineering 37 (2010) 359-370.

440

[24] X. Zhang, G. Ruiz, R. Yu, A new drop-weight impact machine for studying fracture processes in structural concrete, Strain 46(3) (2010) 252-257. 
[25] G. Ruiz, X. Zhang, R. Yu, R. Porras, E. Poveda, J. del Viso, Effect of loading rate on fracture energy of high-strength concrete, Strain 47(6) (2011) $518-524$.

[26] M. Arroyo, M. Ortiz, Local maximum-entropy approximation schemes: a seamless bridge between finite elements and meshfree methods, International Journal for Numerical Methods in Engineering 65 (13) (2006) $2167-2202$.

[27] P. Navas, S. López-Querol, R. Yu, B. Li, B-bar based algorithm applied to meshfree numerical schemes to solve unconfined seepage problems through porous media, International Journal for Numerical and Analytical Methods in Geomechanics 40 (2016) 962-984.

[28] Z. P. Bažant, J. Planas, Fracture and size effect in concrete and other quasi-brittle materials, New directions in Civil Engineering, CRC Press, Boca Raton, Florida, USA, 1998.

[29] N. Beda, J. Ozbolt, A. Sharma, B. Irhan, Dynamic fracture of notched plain concrete beams: 3d finite element study, International Journal of Impact Engineering 77 (2015) 176-188.

[30] R. C. Yu, G. Ruiz, Modeling of static multi-cracking fracture processes in concrete using dynamic relaxation, Computers and Concrete 4 (2004) $371-388$.

[31] R. C. Yu, G. Ruiz, Explicit finite element modeling of static crack propagation in reinforced concrete, International Journal of Fracture 141 (2006) $357-372$.

[32] G. Ruiz, M. Ortiz, A. Pandolfi, Three-dimensional finite-element simulation 
of the dynamic Brazilian tests on concrete cylinders, International Journal for Numerical Methods in Engineering 48 (2000) 963-994. 